\title{
IR Thermography Nondestructive Testing Methods of Composite Materials Used in Aerospace Applications
}

by W. Swiderski

\author{
Military Institute of Armament Technology, Wyszynskiego 7 Str., 05-220 Zielonka, Poland, \\ waldemar.swiderski@wp.pl
}

\begin{abstract}
In case of composite materials we have to deal with the presence of both high mechanical resistance parameters and low specific gravity. Hence in recent years their use rapidly grows in structures for which these characteristics are of paramount importance. There are mainly aerospace structures. Typical defects of composite materials are: delaminating, lack of adhesion, cracks, concentrations and deformations. These defects can come into being both in manufacturing processes and during exploitation. With the development of technology for producing composite materials the requirements are growing for assessment of their quality. For this purpose nondestructive testing (NDT) is used. NDT procedures using IR thermography can be divided into passive and active. For the diagnosis of defects in composite materials of aerospace applications the active IR thermography methods are used in many cases. The paper presents examples of selected results of defects detection and description of used methods.
\end{abstract}

\section{Introduction}

The composite materials have been well-known for thousand years. The notion of composite material (lat. compositus = composite) means material, which is built from at least two different components but their connection is on the macroscopic level.

Currently composites are used in many technologies ranging from implants applied in medicine to cheap construction materials applied in building engineering. In last year's quick development of composite materials is connected with growing demand of aircraft and space and motor industries for light and mechanically resistant materials to replace steel and different metals and their alloys.

For many years aviation has used composite materials, which have very good mechanical properties combined with a relatively low weight. Their use enables construction of very durable and lightweight aircraft structures and reduces maintenance costs. One of advantages of composite using, as opposed to metallic materials, is the possibility of fabrication of more complex shapes in a single process. This reduces number and installation time of elements used in design and number of connecting elements (screws, joins etc.), which are often weak points of construction.

Unfortunately, the excellent mechanical properties of composites decrease significantly when damage occurs. Therefore new composite materials having a higher crack resistance and new methods examining the state of structure in process of their manufacture and use are needed. The infrared radiation used in many investigating methods is a potentially efficient tool for composite testing [1-4].

\section{Specimens}

The most commonly used in aviation are structural sandwich composites and laminated composites.

Materials with honeycomb structure ( honeycomb) belong to a sandwich type composite materials. The structure consists of a core coated on both sides with a thin layer of material coupled to core by connecting material, which typically consists of different kinds of resins. The material of core has a three-dimensional structure consisting of hexagonally shaped cells (Fig.1). Initially, materials of this type, both core and cover was made of aluminum sheet. Such material has a high mechanical strength (for low weight) against bending loads in comparison with solid materials. This characteristic and high resistance to corrosion caused their universal use in aircraft construction and many other applications. There are numbers of failure types which may appear in composites relating both to manufacturing and operating stages. The typical failures, which affect structural integrity and residual strength of the composites, include debonding, delaminating, foreign object inclusions and a kind of damage called as Barely Visible Impact Damage [5-7].

The occurrence of dampness as well as penetration of water into the structure of honeycomb type composite materials testifies to material covering defaults in applications where this material has contact with water or moist air Even small microcracks can make the water or moist air get inside of composite material. In result of temperature changes and condensation of water contained in the air the dampness can appear inside the structure of material. Therefore searching the dampness inside composite material has an important meaning for the evaluation of tightness and defaults in composite material core covering layers. 


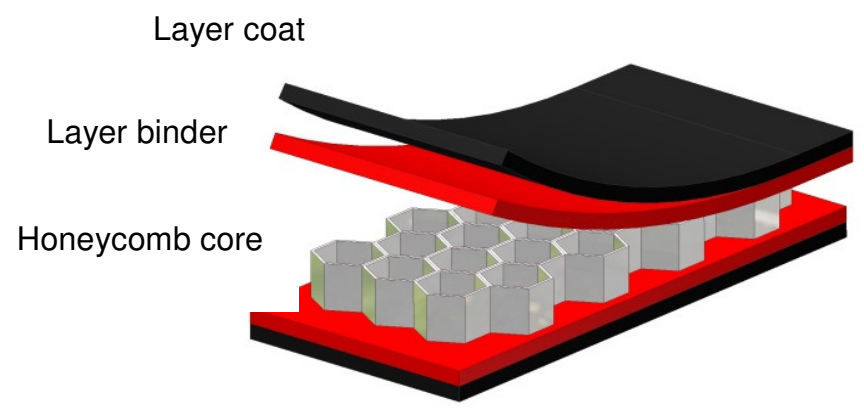

Fig. 1. The structure of honeycomb type of composite material

Nowadays, the extensive use of composites in Aerospace components has become a real fact; for example, in the Boeing 787 Dreamliner aircraft, nearly $50 \%$ of structures have been made of composite materials (Fig. 2) [8].

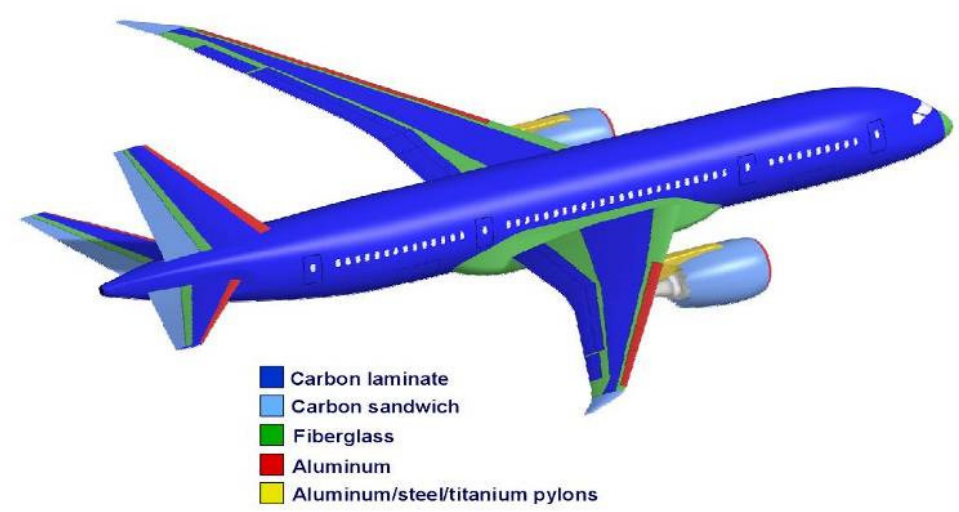

Fig. 2. Example of using composite materials in aircraft construction [8]

In order to get proper estimation and the identification of places in composite material in which the water may appear it was prepared a suitable test - sample. This sample is $290 \times 215 \mathrm{~mm}$ sandwich panel with two $0.7 \mathrm{~mm}$ thick face skins of fiberdux. Between covers the honeycomb core (hexagonal cells) with height $12.8 \mathrm{~mm}$ with areoweb (Fig.3) is placed. Covers are joined with the core by an epoxy resin. In the sample were damaged two internal cells.

The second sample was a piece of a propeller (Fig.4) also being the honeycomb construction of the area $250 \times 300 \mathrm{~mm}$ and thicknesses $38 \mathrm{~mm}$. The core made from the aluminum foil was covered at sides by aluminum sheet $0.5 \mathrm{~mm}$ thickness. There were three defects in the sample: two internal damaged cells of the core and the core with covers joined by a resin.
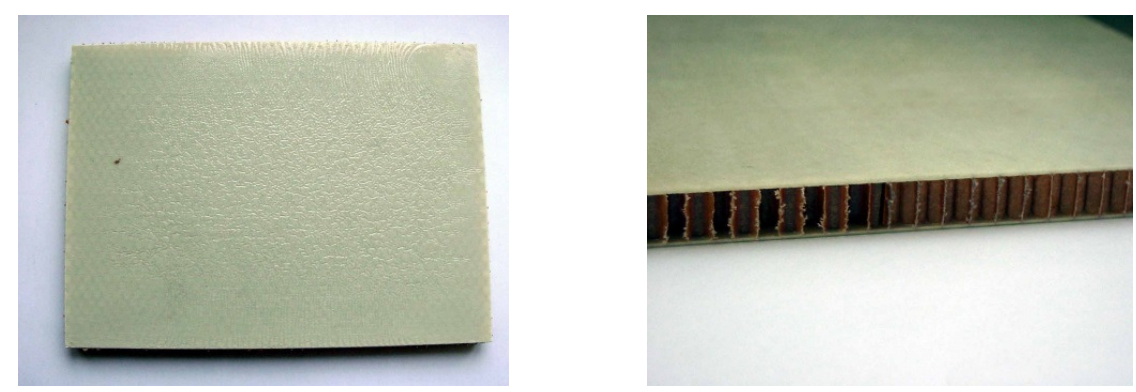

Fig.3. Photography of test samples 

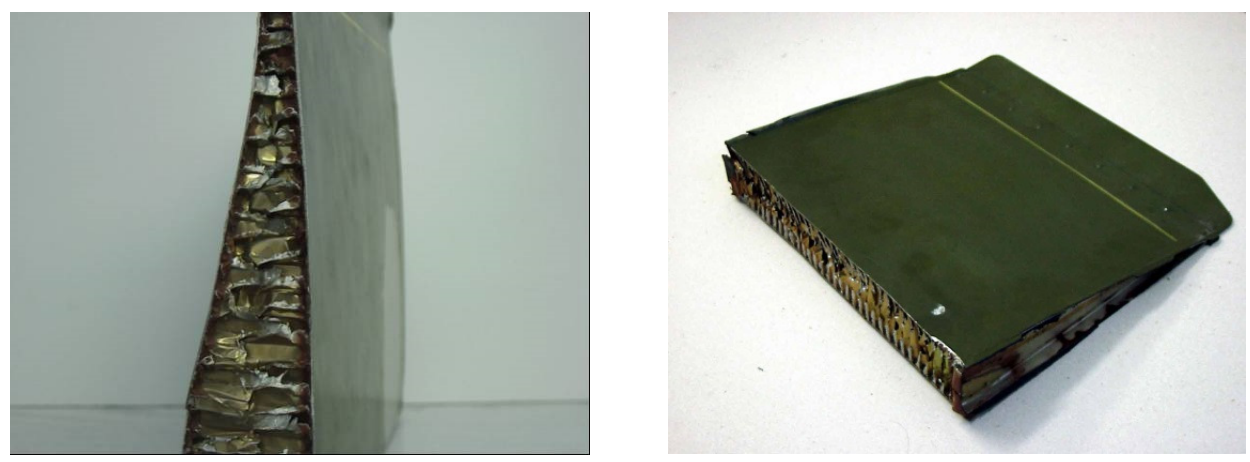

Fig.4. Photography of test sample

\section{Experimental testing}

\subsection{Methods}

In non-destructive testing the interaction of physical fields with structure of tested material is exploited. Within a relatively short development period of modern nondestructive testing the rich domain of technical means came into being or was developed, making possible utilization of very wide range of physical phenomena. Along with data acquisition and data processing technique development the possibilities for deepened analysis of information included in signals representing the impact of physical fields to the structure of testing material have been expanded greatly.

The aim of nondestructive testing by IR thermography is the qualification of object properties during temporary processes occurred at cyclic or impulse thermal stimulation such as heating or cooling applied to a tested object. Available form of answer against thermal stimulation is temperature change and its rate as it contains information about values of thermal capacity and conductivity, characterising internal structure of a tested object. The basic foundation of nondestructive testing methods with IR thermography technics is fact that every class of tested objects reacts in a specific manner against stimulation. There can be different reactions on a particular type of stimulation causing the beginning of thermal wave and reaction of an object against its spreading.

A review of publications on non-destructive testing of aircraft structures shows that methods testing large surfaces [9-11] are most commonly used. Such methods include pulsed and optical lock-in thermography.

In the optical lock - in thermography the stimulation of a thermal wave is achieved by the illumination of the object. The idea of this test is based on the fact that the thermal wave is generated on the whole sample at the same time and is detected and registered separately in the form of amplitude and phase for all pixels simultaneously. The stimulation wave has a sinusoidal shape. In the stationary state the response of the sample is also sinusoidal at the same frequency but with a phase lag that depends on thermal characteristics of the sample. Also the amplitude of the response is distorted what results from the strong attenuation and scattering of the wave in the material of the sample. In fact the phase shift is the most significant for imaging [12-14].

The lock - in thermography system used in our work consists of an infrared camera AGEMA 900 LW, a lock - in module, a controller system and heating lamp (1 kW power) with an infrared filter. FSI FLIR has delivered hardware and software of the system. Both the infrared camera and the heating lamp were located at a distance of about $1 \mathrm{~m}$ from the sample.

Pulsed thermography can be performed in reflection or transmission modes. In this testing a reflection approach was used where IR camera and heating lamp are on the same side of the sample. The lamp (power $3 \mathrm{~kJ}$ ) is positioned $0.5 \mathrm{~m}$ in front of the test sample to heat samples. The lens of the camera FLIR SC 7600 is placed at distance of $0.7 \mathrm{~m}$ from the sample. The sample was heated for $5 \mathrm{~ms}$. The heating and cooling phases were both registered. Results were recorded in the sequence (1600 images) with the frequency $50 \mathrm{kHz}$.

Undesired effects caused by optical heating source (lamp) such as uneven heating of surface of testing sample and noises by state of surface (unevenness, roughness, etc.) are especially visible in the reflection approach. They can make difficult or even impossible detection of subsurface defects especially of deeper locations under surface of the tested object. In order to eliminate or reduce influence of these effects on defects detection there are used such methods of image analysis as: normalizing a sequence, polynomial fitting, Fourier transformation, Principal Component Analysis (PCA), wavelet transformation and dynamic thermal tomography.

Normalizing a sequence - normalization involves the division of each image in the sequence by a chosen normalizing image. Benefit: reducing the phenomenon of uneven heating.

Polynomial fitting - algorithm reduces a sequence of arbitrary length to few images of polynomial coefficients. The restored image sequence is free of high-frequency noise, thus allowing the easy use of such mathematical algorithms as differentiation. 

other pixels.

Correlation - calculating the correlation coefficient between temporal evolutions in a chosen reference point and

Fourier transformation - 1D Fourier transform is applied to pixel-based temporal evolutions of temperature signal in order to produce images of both magnitude and phase at different Fourier frequencies.

Principal Component Analysis - this is a kind of special statistical data treatment which becomes increasingly popular in nondestructive testing. The PCA technique allows converting a source image sequence into the corresponding sequence of the so-called principal components which reflect important statistical relationships between images in the analyzed sequence.

Wavelet transformation - unlike the Fourier transform, the wavelet transform technique allows the determination not only frequency composition of a transient process but also time localizations of particular frequencies. Therefore, it is very efficient in detecting some signal features, in particular jumps and breaks.

Dynamic thermal tomography - With the term "thermal tomography" we specify a procedure that enables slicing a sample by particular depth layers. According to the Thermal NDT theory, this is possible only in a one-sided (frontsurface) test where differential temperature signals, intact with growing defect depth, experience time retardation. Algorithm is intended for producing maxigrams $\left(\Delta T_{m}\right)$ and timegrams $\left(\tau_{m}\right)$, and performing dynamic thermal tomography by selecting the timegrams in particular time intervals [15].

\subsection{Results}

Selected results obtained during testing samples are shown in Figures 5-12. Fig. 5 presents source thermograms obtained by pulsed thermography method after heating a sample (Fig.3). There are visible small traces of two defects located inside composite but at different depths in relation to heating surface. In order to improve imaging several image processing steps were carried out. The effects of these operations are shown in Fig. 7 . It can be seen that a visible correction of defects imaging is only achieved by using PCA.

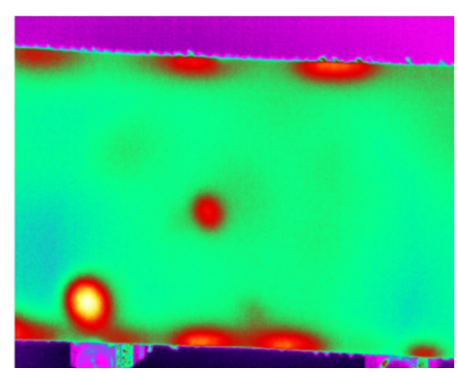

a)

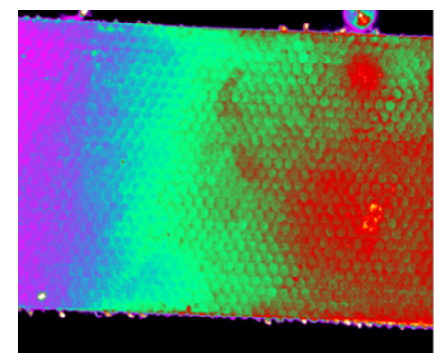

b)

Fig. 5. Source images taken immediately after heating: a) thermogram \#2, b) thermogram\#4

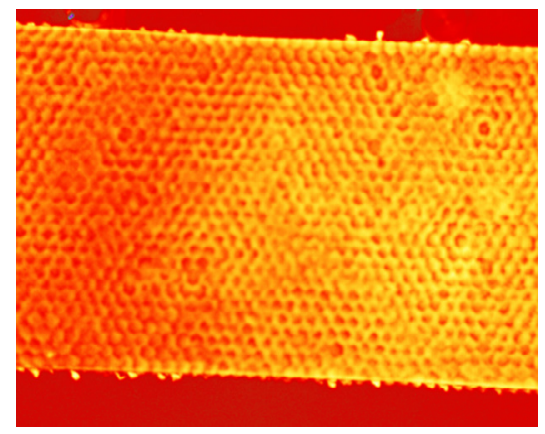

Fig.6. Fourier phasegram ( $4^{\text {th }}$ frequency)

The second sample (Fig. 4) has two defects: detachment of cover from core and second defect is a damaged core cell inside the composite without any direct contact with composite covers. Figures 8 and 9 show results of 
detection of the first defect. Source thermogram recorded immediately after heating by pulsed thermography method is shown in Fig. 8. The improvement of display defect was obtained using Fourier transform and using the wavelet analysis. This can be seen in images of Fig. 9 where wavelets were used by the formula (1) and type of Splash .

$$
\psi(t)=(-1)^{n} \frac{d^{n}}{d t^{n}}\left[e^{-i t} e^{\frac{t^{2}}{2}}\right]
$$



a)

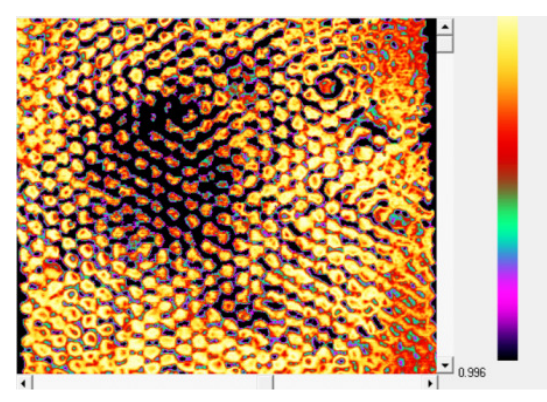

b)

Fig.7. Images after processing: a) PCA component \# 2, b) correlation image

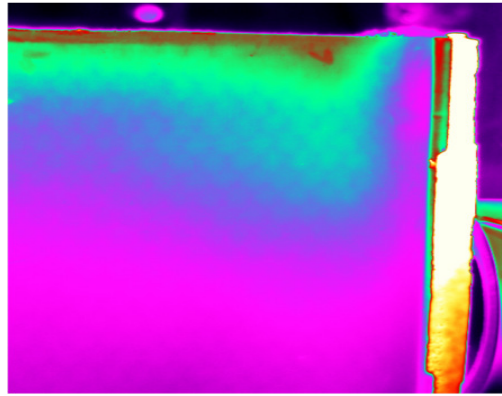

a)

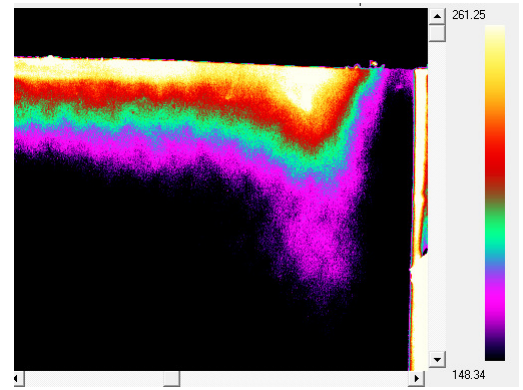

b)

Fig.8. Images of sample Fig.4: source image immediately after heating, b) first defect after Fourier transform

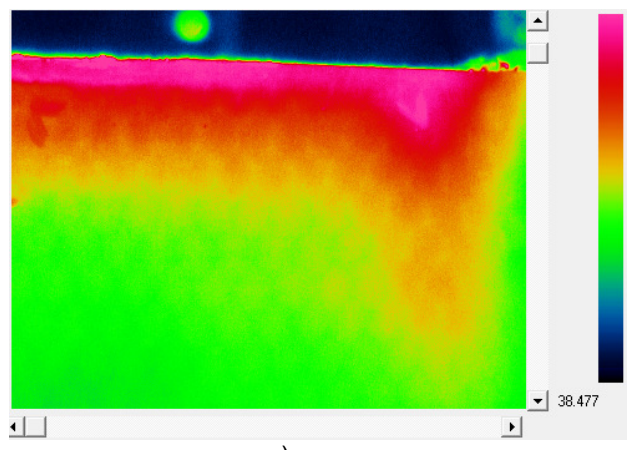

a)

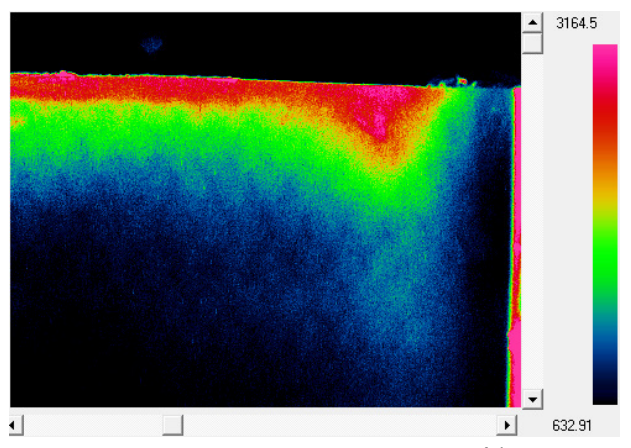

b)

Fig.9. Images of first defect of sample Fig. 4 after wavelet transform: a) formula (1), b) type of Splash

The second defect in the form of coverage detachment from the core is located both by lock-in and pulsed thermography methods. Fig. 10 shows the result obtained by the lock-in method. The best illustration of defect is obtained by using phase image with frequency ca. $0.03 \mathrm{~Hz}$. 
Source image immediately after heating the second defect of sample Fig.4 for pulsed thermography method and after PCA transform processing are shown in Fig. 11. It is seen that the defect is clearly visible after PCA transform.

There is also an improvement of second defect detection after using wavelet transform (Gaussian of order $n=2$ ) and scaled thermogram obtained at the end of recorded sequence. This is shown in Fig. 12.

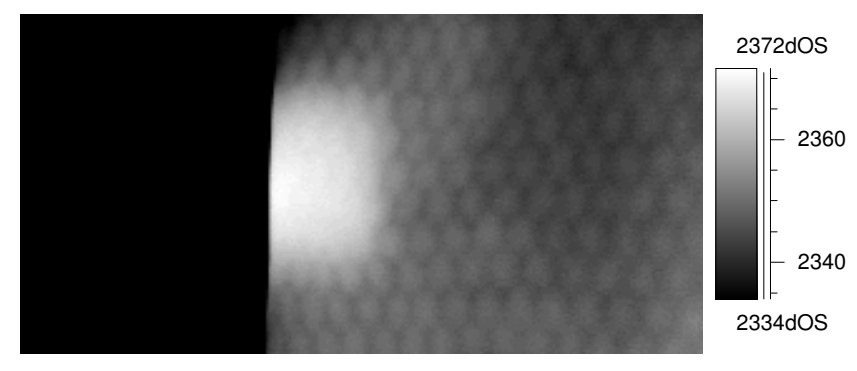

Fig.10. Phase image of second defect for sample Fig.4

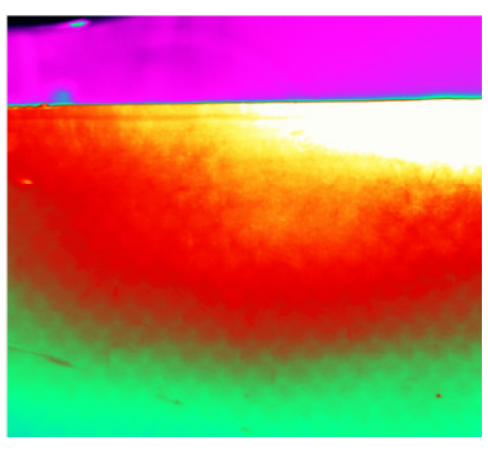

a)

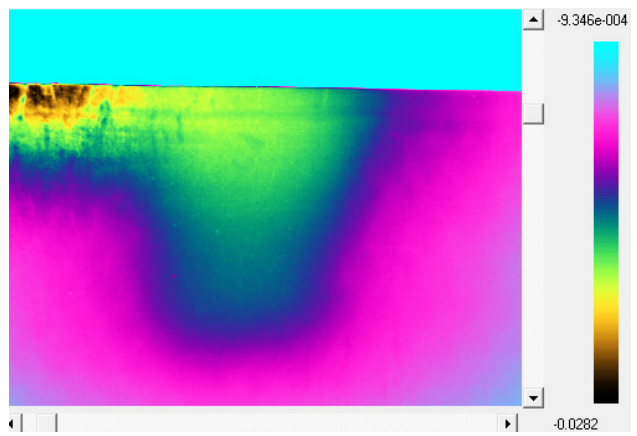

b)

Fig. 11. Images of second defect of sample Fig.4: a) source image immediately after heating, b) PCA component \#2

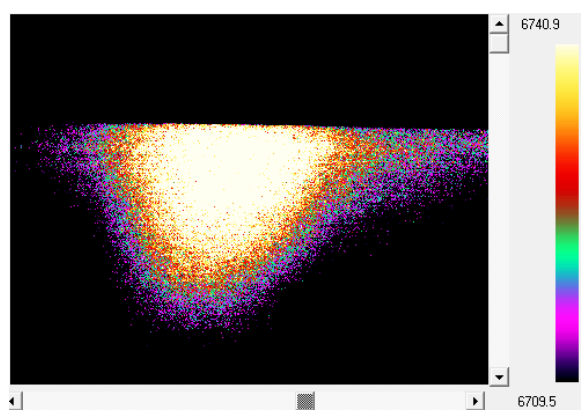

a)

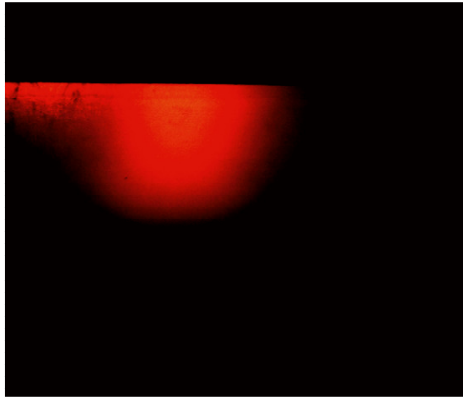

b)

Fig.12. Image of the second defect of sample Fig.4: a) wavelet transform, b) scaled thermogram 


\section{Summary}

This paper describes some diagnostic problems related to the assessment of structural integrity of composites. There is a variety of defects which may appear in composites of honeycomb type. In many cases it is difficult to detect defects such as damaged cells of core inside of the composite covered by a thin metal plate used in aircraft components. However, this is rarely occurring case of internal damaged cells without outside visible effects of damage. The occurrence of visible damage on outside surface of the composite makes easy the location of internal damaged structure.

Selected results received from thermography testing showed that in some cases the image processing can greatly improve efficiency of detection defects of honeycomb composites. However, it cannot be specified directly which image processing method is more effective. It is because it depends on individual cases of damage, testing condition and used thermography methods.

\section{Acknowledgements}

The help of Prof. V. Vavilov is thankfully appreciated.

\section{REFERENCES}

[1] Maldague X. P. V. "Theory and practice of infrared technology for non-destructive testing" John Wiley\&Sons, Inc. New York, 2001

[2] Meola C., Carlomagno G. M., Bonavolontà C., Valentino M., "Monitoring Composites under Bending Tests with Infrared Thermography". Advances in Optical Technologies, Volume 2012 (2012), 7 pages

[3] Meola C., Carlomagno G. M., "Infrared thermography in non-destructive inspection: theory and practice," Recent Advances in Non Destructive Inspection, C. Meola, Ed., Nova Science, New York, NY, USA, pp. 89123,2010

[4] Garnier C., Lorrain B., Pastor M-L., "Impact damage evolution under fatigue loading by InfraRed Thermography on composite structures". EPJ Web of Conferences, published by EDP Sciences, 2010, pages 8

[5] Dragan, K., Klimaszewski, S. "Holistic Approach for Structural Integrity Evaluation of Composite Main Rotor Blades"WCNDT 2008, Shanghai, China.

[6] Findeis, D.: "The feasibility of Optical Interference -based NDE Methods to Inspect Helicopter Rotor Blades", Dept. of Mechanical Eng. Univ. of Cape Town, 1999.

[7] Capriotti, R. "NDT Techniques for the Evaluation of Impact Damage on Aeronautical Structures, Aeronautica Militare Italiana", WCNDT 2000, Roma, Italy.

[8] Roach D., DiMambro J., Enhanced Inspection Methods to Characterize Bonded Joints: Moving Beyond Flaw Detection to Quantify Adhesive Strength, Air Transport Association Nondestructive Testing Forum, Forth Worth (USA) 17 - 19.10. 2006.

[9] Capriotti R., Dati E., Silvestri P., Trivisonno G., Marinetti S., Dal Toc S., Bison P. G., and Grinzato E., "NDT Techniques for the Evaluation of Impact Damage on Aeronautical Structure", 15 $5^{\text {th }}$ WCNDT, on CD, Roma 2000

[10] Garcia J., Hernandez N., Morales A. and Servent R., "Considerations of thermographic inspection reliability of aircraft components" $8^{\text {th }}$ ECNDT, on CD, Barcelona 2002.

[11] Wu D., Salerno A., Malter A., Aoki R., Kochendorfer R., Kachele P. K., Woithe K., Pfister K., Busse G., "Inspection of aircraft structural components using lockin - thermography" QIRT, Stuttgart , 1996 , p 251-256.

[12] Swiderski W. "Lock-in Thermography to rapid evaluation of destruction area in composite materials used in military applications" SPIE vol. 5132, 2003, pp. 506-517

[13] Busse G., Gleiter A., Spiessberger C., "NDE using Lockin-Thermography: principle and recent developments". Poroceedings of NDTMS-2011, Part 1, Istanbul, Springer, 2013, pp.627-632.

[14] Swiderski W., Szabra D., Wojcik J., „Nondestructive evaluation of aircraft components by thermography using different heat sources" Qutitative InfraRed Thermography 6, Dubrovnik 2002, pp. 79-84.

[15] ThermoLab ${ }^{T M}$, Operation Manual, Innovation Ltd. 2013 